\title{
Students' perceptions of online discussions, participation and e-moderation behaviours in peer-moderated asynchronous online discussions
}

\begin{abstract}
The aim of this study was to investigate the relationships between students' perceptions of online discussions, participation and e-moderation behaviours in peer-moderated asynchronous online discussions. Using survey data, the learning system's activity log and discussion transcripts, the authors analysed how 84 learners' perceptions of online discussions influenced their participation and e-moderation behaviours. The results showed that Cognitive and Skill II (critical thinking skills) perceptual aspects significantly influenced the quantity posting and participation patterns. Meanwhile, Skill I (reading and writing skills) and Efficacy were critical factors that influenced quantity non-posting participation. It was also found that the perceptual aspects significantly influenced learners' e-moderation behaviours to the extent that Affection and Efficacy were critical factors that influenced lower-level e-moderation supports, while Cognition, Affection and Skill II were critical factors that influenced higher-level e-moderation supports. All e-moderation supports were found to be correlated with peers' quantity posting and participation patterns, except for Access and motivation support.
\end{abstract}

Keyword: Perceptions; Participation; Peer-moderated asynchronous online discussions; Emoderation behaviours 